Research Article

\title{
Estimation of Constant Stress Partially Accelerated Life Test for Fréchet Distribution with Type-I Censoring
}

\author{
Abdullah Ali H. Ahmadini $\left(\mathbb{D},{ }^{1}\right.$ Wali Khan Mashwani $\left(\mathbb{D},{ }^{2}\right.$ Rehman Ahmad Khan Sherwani, ${ }^{3}$ \\ Shokrya S. Alshqaq, ${ }^{1}$ Farrukh Jamal $\mathbb{D},{ }^{4}$ Miftahuddin Miftahuddin $\mathbb{D},{ }^{5}$ Kamran Abbas, ${ }^{6}$ \\ Faiza Razaq, ${ }^{6}$ Mohammed Elgarhy $\mathbb{D}^{7},{ }^{7}$ and Sanaa Al-Marzouki ${ }^{8}$ \\ ${ }^{1}$ Department of Mathematics, Faculty of Science, Jazan University, Jazan, Saudi Arabia \\ ${ }^{2}$ Institute of Numerical Sciences, Kohat University of Science \& Technology, Kohat 26000, Pakistan \\ ${ }^{3}$ Collerge of Statistical and Actuarial Sciences, University of the Punjab, Lahore, Pakistan \\ ${ }^{4}$ Department of Statistics, The Islamia University of Bahawalpur, Bahawalpur 61300, Pakistan \\ ${ }^{5}$ Department of Statistics, Faculty of Mathematics and Sciences, Syiah Kuala University, Banda Aceh, Indonesia \\ ${ }^{6}$ Department of Statistics, University of Azad Jammu and Kashmir, Muzaffarabad, Pakistan \\ ${ }^{7}$ The Higher Institute of Commercial Sciences, Al Mahalla Al Kubra, Algarbia 31951, Egypt \\ ${ }^{8}$ Statistics Department, Faculty of Science, King Abdul Aziz University, Jeddah 21551, Saudi Arabia
}

Correspondence should be addressed to Miftahuddin Miftahuddin; miftah@unsyiah.ac.id

Received 3 April 2021; Revised 22 April 2021; Accepted 30 April 2021; Published 12 May 2021

Academic Editor: Dr. Dilbag Singh

Copyright ( $) 2021$ Abdullah Ali H. Ahmadini et al. This is an open access article distributed under the Creative Commons Attribution License, which permits unrestricted use, distribution, and reproduction in any medium, provided the original work is properly cited.

\begin{abstract}
Modern reliability engineering accelerated life tests (ALT) and partially accelerated life tests (PALT) are widely used to obtain the timely information on the reliability of objects, products, elements, and materials as well as to save time and cost. The ALTs or PALTs are useful in determining the failed manners of the items at routine conditions by using the information of the data generated from the experiment. PALT is the most sensible method to be used for estimating both ordinary and ALTs. In this research, constant stress PALT design for the Fréchet distribution with type-I censoring has been investigated due to a wide applicability of the Fréchet distribution in engineering problems especially in hydrology. The distribution parameters and acceleration factor are obtained by using the maximum likelihood method. Fisher's information matrix is used to develop the asymptotic confidence interval estimates of the model parameters. A simulation study is conducted to illustrate the statistical properties of the parameters and the confidence intervals by using the $\mathrm{R}$ software. The results indicated that the constant stress PALT plan works well. Moreover, a numerical example is given to exemplify the performance of the proposed methods.
\end{abstract}

\section{Introduction}

The continuous progress in manufacturing design makes the products and materials highly reliable [1]. It is becoming more and more difficult to get information under normal use conditions about the lifespan of objects and materials with higher reliability at the testing time, and it becomes very expensive and time-consuming under such conditions [2]. The sample is examined for these objects at more serious circumstances than usual [3]. These situations belong to stresses, which may be in the form of voltage, force, temperature, vibrations, moisture, etc [4-7]. To develop a new life testing method, these problems have motivated the researcher to get information timely on the reliability of components, objects, and products.

Particularly, when test items are run at both normal and higher than normal stress conditions, the more suitable test to be applied is the partially accelerated life test (PALT) [8-10]. Furthermore, it is the most important method to be utilized for evaluating the acceleration factor and thus extrapolating the accelerated test data to use condition. The PALT combines both accelerated and ordinary life tests. 
Thus, PALT is reasonable for estimating the acceleration factor $(\beta>1)$, "which is the ratio of the hazard rate at the accelerated condition to that at normal use conditions" [11].

The commonly used methods in PALT are constant stress PALT (CSPALT) [4, 12, 13], step stress PALT (SSPALT) [5, 14-16], and progressive stress PALT (PSPALT) [17-19]. In CSPALT, products are examined at either accelerated condition or at normally used condition before the test is being terminated.

Additionally, censoring is very ordinary in life testing experiments. It frequently happens that the experiment is censored when the experimenter may not be able to examine the lifespan of all units put on the test because of time restrictions and other limitations on the data collection. It is usually used when a distribution of exact lifetimes is known only for a part of the test items, and the remainder of the lifetimes is known only to exceed certain values under a life test. The two most common types of censoring are type-I censoring (time censoring) and type-II censoring (failure censoring).

For a brief overview of PALT, literature is abundant on designing PALT. Bai and Chung $[8,9]$ discussed both the problem of estimation and optimally designing constant stress PALT for exponential and lognormal distributions. Ismail [20] has applied maximum likelihood (ML) and Bayesian methods for evaluating the parameters of Pareto distribution of the second kind and the acceleration factor. Ismail et al. [21] considered the case of CSPALT when under type-I censoring two stress levels was included. Similarly, Cheng and Wang [22], Ismail [23], Kamal et al. [13], Wang $[24,25]$, and Hassan et al. [12] applied PALT and acceleration factors using different lifetime distributions; PALT and CSPALT have been studied via Bayesian approach $[19,26-28]$.

In this paper, the CSPALT plan using type-I censoring with the assumption that the lifetimes of the test items at use condition follow Fréchet distribution which is considered. Maurice Fréchet was a French mathematician who had identified one possible limit distribution for the largest order statistic in 1927 [29]. The probability density function (PDF) of Fréchet distribution is

$$
f(t)=\frac{\alpha}{\theta}\left(\frac{t}{\theta}\right)^{-(\alpha+1)} \exp \left(-\left(\frac{t}{\theta}\right)^{-\alpha}\right), \quad t, \alpha, \theta>0,
$$

where $\alpha$ is the shape parameter and $\theta$ is the scale parameter. This distribution has a heavy upper tail and is bounded on the lower side $(t>0)$. The cumulative distribution function $(\mathrm{CDF})$ is

$$
F(t)=\exp \left[-\left(\frac{t}{\theta}\right)^{-\alpha}\right], \quad t, \alpha, \theta>0,
$$

The reliability function of Fréchet distribution is

$$
R(t)=1-\exp \left[-\left(\frac{t}{\theta}\right)^{-\alpha}\right], \quad t, \alpha, \theta>0
$$

The Fréchet distribution is applied to extreme events and linked to the modeling of several real-world phenomena, including human lifetimes, flood and seismic analyses, radioactive emissions, and maximum one-day rainfalls.

The rest of the study is arranged as follows. In Section 2, the assumption and test procedures for Fréchet distribution with constant stress using type-I censoring are discussed. The maximum likelihood estimates (MLEs) of the distribution parameters and acceleration factor are presented in Section 3. Fisher information matrix and asymptotic confidence interval are discussed in Section 4. A simulation study is presented in Section 5. Finally, Section 6 presents the results, conclusion, and recommendations for future work.

\section{Model Description}

The following notations are used for model description:

(i) $n=$ total number of objects in a PALT

(ii) $\tau=$ censoring time of CSPALT

(iii) $t=$ lifespan of an object at normal use condition

(iv) $X=$ lifespan of an object at accelerated condition

(v) $\beta=$ acceleration factor $(\beta>1)$ and defined as $\beta=T /$ $X$

(vi) $t_{i}=$ observed lifetime of $i^{\text {th }}$ object at usual use condition

(vii) $x_{j}=$ observed lifetime of $j^{\text {th }}$ unit at accelerated condition

(viii) $t_{(1)} \leq \ldots \leq t_{\left(n_{u}\right)} \leq \tau=$ ordered failure time at use condition

(ix) $x_{(1)} \leq \ldots \leq x_{\left(n_{a}\right)} \leq \tau=$ ordered failure time at accelerated condition

2.1. Test Procedure of CSPALT. In CSPALT, total test units " $n$ " are randomly divided into two samples of sizes " $n r$ " and $n(1-r)$, respectively, where " $r$ " is the sample proportion. In the first sample, " $n r$ " items are randomly chosen among " $n$ " items, which are assigned to accelerated conditions and the other $n(1-r)$ are assigned to regular use conditions. Each test object of every sample is run until the censoring time " $\tau$ " and the test condition is not changed.

2.2. Assumptions. The following assumptions are also made for CSPALT.

(i) Under normal-use condition, lifespan of an object supports Fréchet distribution.

(ii) The lifespan of an object at accelerated condition is attained by $X=\beta^{-1} T$, where $\beta>1$, which is an acceleration factor. Therefore, the PDF at accelerated condition is

$$
f(x, \alpha, \beta, \theta)=\frac{\alpha \beta}{\theta}\left(\frac{\beta x}{\theta}\right)^{-(\alpha+1)} \exp \left[-\left(\frac{\beta x}{\theta}\right)^{-\alpha}\right], \quad x, \theta, \beta>0 .
$$


(iii) The lifespan $T_{i}, i=1,2, \ldots, n(1-r)$ of objects allocated to the normal condition are independently and identically distributed (i.i.d) random variables.

(iv) The lifespan $X_{j}, j=1,2, \ldots, n r$ of objects assigned to accelerate are i.i.d. random variables.

(v) The lifetimes $T_{i}$ and $X_{j}$ are mutually independent.

\section{Parameter Estimation}

The ML method is one of the most important and widely used methods in statistics. The main reasons are that the ML method is very vigorous and provides the estimates of parameters with good statistical properties such as consistency, asymptotic unbiased, asymptotic efficiency, and asymptotic normality.

Let $\delta_{u i}$ and $\delta_{a j}$ be the indicator functions under the use and accelerated conditions, respectively, such that

$$
\begin{aligned}
& \delta_{u i}=\left\{\begin{array}{ll}
1, & t_{i} \leq \tau, \\
0, & \text { otherwise, }
\end{array} \quad i=1,2, \ldots, n(1-r),\right. \\
& \delta_{a j}=\left\{\begin{array}{ll}
1, & x_{j} \leq \tau, \\
0, & \text { otherwise, }
\end{array} \quad j=1,2, \ldots, n r .\right.
\end{aligned}
$$

The likelihood function for $\left(t_{i}, \delta_{u i}\right)$ and $\left(x_{j}, \delta_{a j}\right)$ can be expressed as

$$
\begin{aligned}
L_{u i}\left(t_{i}, \delta_{u i} \mid \alpha, \theta\right) & =\prod_{i=1}^{n(1-r)}\left[\frac{\alpha}{\theta}\left(\frac{t_{i}}{\theta}\right)^{-(\alpha+1)} \exp \left(-\left(\frac{t_{i}}{\theta}\right)^{-\alpha}\right)\right]^{\delta_{u i}} \cdot\left[1-\exp \left(-\left(\frac{\tau}{\theta}\right)^{-\alpha}\right)\right]^{\bar{\delta}_{u i}}, \\
L_{a j}\left(x_{j}, \delta_{a j} \mid \alpha, \beta, \theta\right) & =\prod_{j=1}^{n r}\left[\frac{\alpha \beta}{\theta}\left(\frac{\beta x_{j}}{\theta}\right)^{-(\alpha+1)} \exp \left(-\left(\frac{\beta x_{j}}{\theta}\right)^{-\alpha}\right)\right]^{\delta_{a j}} \times\left[1-\exp \left(-\left(\frac{\beta \tau}{\theta}\right)^{-\alpha}\right)\right]^{\bar{\delta}_{a j}},
\end{aligned}
$$

where $\bar{\delta}_{u i}=1-\delta_{u i}$ and $\bar{\delta}_{a j}=1-\delta_{a j}$.

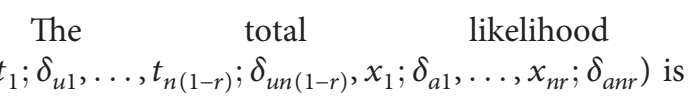

$$
\begin{aligned}
L(t, x \mid \alpha, \beta, \theta)= & L_{u i}\left(t_{i}, \delta_{u i} \mid \alpha, \theta\right) * L_{a j}\left(x_{j}, \delta_{a j} \mid \alpha, \beta, \theta\right) \\
L(t, x \mid \alpha, \beta, \theta)= & \prod_{i=1}^{n(1-r)}\left[\frac{\alpha}{\theta}\left(\frac{t_{i}}{\theta}\right)^{-(\alpha+1)} \exp \left(-\left(\frac{t_{i}}{\theta}\right)^{-\alpha}\right)\right]^{\delta_{u i}}\left[1-\exp \left(-\left(\frac{\tau}{\theta}\right)^{-\alpha}\right)\right]^{\bar{\delta}_{u i}} \times \prod_{j=1}^{n r}\left[\frac{\alpha \beta}{\theta}\left(\frac{\beta x_{j}}{\theta}\right)^{-(\alpha+1)} \exp \left(-\left(\frac{\beta x_{j}}{\theta}\right)^{-\alpha}\right)\right]^{\delta_{a j}} \\
& \times\left[1-\exp \left(-\left(\frac{\beta \tau}{\theta}\right)^{-\alpha}\right)\right]^{\bar{\delta}_{a j}} .
\end{aligned}
$$

It is more convenient to work with the log-likelihood function. The log-likelihood function of (8) is

$$
\begin{aligned}
\ell= & \ln L=\sum_{i=1}^{n(1-r)} \delta_{u i}\left[\ln \alpha-\ln \theta-(\alpha+1) \ln \left(\frac{t_{i}}{\theta}\right)-\left(\frac{t_{i}}{\theta}\right)^{-\alpha}\right]+\sum_{i=1}^{n(1-r)} \bar{\delta}_{u i} \ln \left[1-\exp \left(-\left(\frac{\tau}{\theta}\right)^{-\alpha}\right)\right] \\
& +\sum_{j=1}^{n r} \delta_{a j}\left[\ln \alpha+\ln \beta-\ln \theta-(\alpha+1) \ln \left(\frac{\beta x_{j}}{\theta}\right)-\left(\frac{\beta x_{j}}{\theta}\right)^{-\alpha}\right]+\sum_{j=1}^{n r} \bar{\delta}_{a j} \cdot \ln \left[1-\exp \left(-\left(\frac{\beta \tau}{\theta}\right)^{-\alpha}\right)\right] .
\end{aligned}
$$

Let $n_{u}$ and $n_{a}$ be the numbers of items that failed at normal and accelerated conditions, respectively. Similarly, let $c_{u}$ and $c_{a}$ be the numbers of items censored at normal and accelerated conditions, respectively: 


$$
\begin{aligned}
& \sum_{i=1}^{n(1-r)} \delta_{u i}=n_{u} \\
& \sum_{j=1}^{n r} \delta_{a j}=n_{a} \\
& \sum_{i=1}^{n(1-r)} \bar{\delta}_{u i}=c_{u} \\
& \sum_{i=1}^{n(1-r)} \bar{\delta}_{u i}=c_{a} \\
& l=n_{u} \ln \alpha-n_{u} \ln \theta-(\alpha+1) \sum_{i=1}^{n(1-r)} \delta_{u i} \ln \left(\frac{t_{i}}{\theta}\right)-\sum_{i=1}^{n(1-r)} \delta_{u i}\left(\frac{t_{i}}{\theta}\right)^{-\alpha}+c_{u} \cdot \ln \left[1-\exp \left(-\left(\frac{\tau}{\theta}\right)^{-\alpha}\right)\right] \\
& +n_{a} \ln \alpha+n_{a} \ln \beta-n_{a} \ln \theta-(\alpha+1) \sum_{j=1}^{n r} \delta_{a j} \ln \left(\frac{\beta x_{j}}{\theta}\right)-\sum_{j=1}^{n r} \delta_{a j}\left(\frac{\beta x_{j}}{\theta}\right)^{-\alpha}+c_{a} \cdot \ln \left[1-\exp \left(-\left(\frac{\beta \tau}{\theta}\right)^{-\alpha}\right)\right] \text {, } \\
& l=\left(n_{u}+n_{a}\right) \ln \alpha-\left(n_{u}+n_{a}\right) \ln \theta+n_{a} \ln \beta-(\alpha+1) \sum_{i=1}^{n(1-r)} \delta_{u i} \ln \left(\frac{t_{i}}{\theta}\right)-\sum_{i=1}^{n(1-r)} \delta_{u i}\left(\frac{t_{i}}{\theta}\right)^{-\alpha}+c_{u} \\
& * \ln \left[1-\exp \left(-\left(\frac{\tau}{\theta}\right)^{-\alpha}\right)\right]-(\alpha+1) \sum_{j=1}^{n r} \delta_{a j} \ln \left(\frac{\beta x_{j}}{\theta}\right)-\sum_{j=1}^{n r} \delta_{a j}\left(\frac{\beta x_{j}}{\theta}\right)^{-\alpha}+c_{a} \ln \left[1-\exp \left(-\left(\frac{\beta \tau}{\theta}\right)^{-\alpha}\right)\right] \text {. }
\end{aligned}
$$

MLEs of $\alpha, \theta$, and $\beta$ are obtained by solving the system of equations $(\partial l / \partial \alpha)=0,(\partial l / \partial \theta)=0$ and $(\partial l / \partial \beta)=0$, respectively:

$$
\begin{aligned}
\frac{\partial l}{\partial \alpha}= & \frac{\left(n_{u}+n_{a}\right)}{\alpha}-\sum_{i=1}^{n(1-r)} \delta_{u i} \ln \left(\frac{t_{i}}{\theta}\right)+\sum_{i=1}^{n(1-r)} \delta_{u i}\left(\frac{t_{i}}{\theta}\right)^{-\alpha} \ln \left(\frac{t_{i}}{\theta}\right)-\frac{c_{u}(\tau / \theta)^{-\alpha} \ln (\tau / \theta) e^{-(\tau / \theta)^{-\alpha}}}{1-e^{-(\tau / \theta)^{-\alpha}}} \\
& -\sum_{j=1}^{n r} \delta_{a j} \ln \left(\frac{\beta x_{j}}{\theta}\right)+\sum_{j=1}^{n r} \delta_{a j}\left(\frac{\beta x_{j}}{\theta}\right)^{-\alpha} \ln \left(\frac{\beta x_{j}}{\theta}\right)-\frac{c_{a}(\beta \tau / \theta)^{-\alpha} \ln (\beta \tau / \theta) e^{-(\beta \tau / \theta)^{-\alpha}}}{1-e^{-(\beta \tau / \theta)^{-\alpha}}} \\
\frac{\partial l}{\partial \theta}= & \left(n_{u}+n_{a}\right) \frac{\alpha}{\theta}+c_{u}\left(\frac{\alpha \theta^{\alpha-1} \tau^{-\alpha} e^{-(\tau / \theta)^{-\alpha}}}{1-e^{-(\tau / \theta)^{-\alpha}}}\right)+c_{a}\left(\frac{\alpha \theta^{\alpha-1}(\beta \tau)^{-\alpha} e^{-(\beta \tau / \theta)^{-\alpha}}}{1-e^{-(\beta \tau / \theta)^{-\alpha}}}\right) \\
& -\alpha \theta^{\alpha-1}\left(\sum_{i=1}^{n(1-r)} \delta_{u i}\left(t_{i}\right)^{-\alpha}+\beta^{-\alpha} \sum_{j=1}^{n r} \delta_{a j}\left(x_{j}\right)^{-\alpha}\right), \\
\frac{\partial l}{\partial \beta}= & -n_{a}\left(\frac{\alpha}{\beta}\right)+\alpha \theta^{\alpha} \beta^{-(\alpha+1)} \sum_{j=1}^{n r} \delta_{a j} x_{j}^{-\alpha}-c_{a}\left(\frac{\alpha(\beta \tau / \theta)^{-\alpha} e^{-(\beta \tau / \theta)^{-\alpha}}}{\beta\left(1-e^{-(\beta \tau / \theta)^{-\alpha}}\right)}\right) .
\end{aligned}
$$

The above equations cannot be written in the closed form. So, an iterative procedure can be used to get MLEs. Here, we use the BFGS Quasi-Newton Optimization method which is available in the $\mathrm{R}$ software. Furthermore, for interval estimates of model parameters, we need the Fisher information matrix.
3.1. Fisher Information Matrix. Here, it is hard to obtain an explicit solution to nonlinear equations, so an iterative method such as the Newton Raphson method [30] is used to find the solution of equations to obtain MLEs. Also, we get the asymptotic confidence intervals. Hence, the asymptotic 
variance of the MLE can be attained by the inverse of the observed Fisher information matrix:

$$
V=\left(\begin{array}{ccc}
A \operatorname{Var}(\widehat{\alpha}) & A \operatorname{Cov}(\widehat{\alpha} \widehat{\theta}) & A \operatorname{Cov}(\widehat{\alpha} \widehat{\beta}) \\
A \operatorname{Cov}(\widehat{\theta} \widehat{\alpha}) & A \operatorname{Var}(\widehat{\theta}) & A \operatorname{Cov}(\widehat{\theta} \widehat{\beta}) \\
A \operatorname{Cov}(\widehat{\beta} \widehat{\alpha}) & A \operatorname{Cov}(\widehat{\beta} \widehat{\theta}) & A \operatorname{Var}(\widehat{\beta})
\end{array}\right) .
$$

The $100(1-\alpha)$ percent asymptotic confidence interval for $\alpha, \theta$, and $\beta$ can be written as

$$
\begin{aligned}
& {\left[\widehat{\alpha} \pm Z_{(1-\alpha)} \sqrt{A \operatorname{Var}(\widehat{\alpha})}\right]} \\
& {\left[\widehat{\theta} \pm Z_{(1-\alpha)} \sqrt{A \operatorname{Var}(\widehat{\theta})}\right]} \\
& {\left[\widehat{\beta} \pm Z_{(1-\alpha)} \sqrt{A \operatorname{Var}(\widehat{\beta})}\right]}
\end{aligned}
$$

respectively.

\section{Simulation Study}

A simulation study is carried out to evaluate the performance of proposed estimators. It is performed by using the $\mathrm{R}$ software (3.2.3) for demonstrating the theoretical outcomes of the estimation problems. The performances of MLEs are evaluated through mean square error (MSE) and absolute bias of estimates. Also, $90 \%$ and $95 \%$ confidence limits are constructed for parameters and acceleration factors. For this purpose, several data sets are generated from two-parameter Fréchet distribution with sizes $n=50,100,150,200,300$, and 500. Samples are generated by inverse transformation of the Fréchet distribution and the process is replicated 1000 times.

The simulation steps are summarized below:

(1) For estimation, the sample size is varied to see the effect of large and small samples are considered. The random samples are generated from Fréchet distribution by using transformation $x=\theta(-\log (u))^{-1 / \alpha}$, where $u$ is uniformly distributed, i.e., $U \sim$ uniform $(0$, 1).

(2) The values for true parameters are taken as $(\alpha=0.75$, $\beta=1.2, \theta=1), \quad(\alpha=1, \beta=1.1, \theta=1)$, and $(\alpha=0.8$, $\beta=1.2, \theta=0.8)$, respectively. The selected censoring level is $0.7 * n(1-r)$ and $0.3 * n r$.

(3) The " $n$ " test item is split with sample proportion $r=0.3$, such that " $n r$ " items are assigned at the accelerated condition and $n(1-r)$ are allotted to normal use condition.

(4) The estimates of the model parameters and their corresponding summary statistics are obtained by the CSPALT model.

(5) For the different combination of stresses, the MLEs for $\alpha, \beta$, and $\theta, \mathrm{MSE}$, and absolute bias of the estimates and ninety percent and ninety-five percent CIs

\begin{tabular}{|c|c|c|c|c|c|}
\hline$\eta$ & Parameters & Estimates & MSE & $\begin{array}{c}\text { Absolute } \\
\text { bias }\end{array}$ & $95 \% \mathrm{CI}$ \\
\hline \multirow{3}{*}{50} & $\alpha$ & 1.5870 & 0.8109 & 0.8370 & $\begin{array}{c}-0.8226 \\
3.9966\end{array}$ \\
\hline & $\beta$ & 1.1830 & 0.1229 & 0.0169 & $\begin{array}{c}0.49494 \\
1.8711\end{array}$ \\
\hline & $\theta$ & 0.4159 & 0.3485 & 0.5840 & $\begin{array}{c}-1.2117 \\
2.0436 \\
\end{array}$ \\
\hline \multirow{3}{*}{100} & $\alpha$ & 1.4755 & 0.5836 & 0.7352 & \multirow{3}{*}{$\begin{array}{c}-0.5724, \\
3.5234 \\
0.7797,1.7473 \\
-1.2112, \\
2.0408\end{array}$} \\
\hline & $\beta$ & 1.2635 & 0.0715 & 0.0531 & \\
\hline & $\theta$ & 0.4147 & 0.3465 & 0.5858 & \\
\hline \multirow{3}{*}{150} & $\alpha$ & 1.4603 & 0.53274 & 0.7103 & \multirow{3}{*}{$\begin{array}{c}-0.5569, \\
3.5019 \\
0.8076,1.7464 \\
-1.2172 \\
2.0429\end{array}$} \\
\hline & $\beta$ & 1.2914 & 0.04893 & 0.0776 & \\
\hline & $\theta$ & 0.4136 & 0.34611 & 0.5864 & \\
\hline \multirow{3}{*}{200} & $\alpha$ & 1.4386 & 0.49254 & 0.68863 & \multirow{3}{*}{$\begin{array}{c}-0.5043, \\
3.3997 \\
0.8576,1.7275 \\
-1.2125 \\
2.0402\end{array}$} \\
\hline & $\beta$ & 1.2992 & 0.04160 & 0.09918 & \\
\hline & $\theta$ & 0.4126 & 0.34663 & 0.58743 & \\
\hline \multirow{3}{*}{300} & $\alpha$ & 1.4221 & 0.46364 & 0.67210 & \multirow{3}{*}{$\begin{array}{c}-0.4592, \\
3.3088 \\
0.8753,1.7414 \\
-1.2159 \\
2.0416\end{array}$} \\
\hline & $\beta$ & 1.3089 & 0.03655 & 0.10886 & \\
\hline & $\theta$ & 0.4128 & 0.34592 & 0.58724 & \\
\hline
\end{tabular}
are derived.
TABle 1: Average estimates of CSPLAT with $\alpha=0.75, \beta=1.2, \theta=1$, and $r=0.3$.

(6) Tables 1-3 summarize the results of the ML estimates for $\alpha, \beta$, and $\theta$. The numerical results presented in Table 4 are based on 1000 simulation replications.

(7) Figures 1-3 present the MSEs against different sample sizes for the MLEs of $\alpha, \beta$, and $\theta$.

\section{Numerical Example}

For illustration, we assume that the values of Fréchet constant stress model parameters are known and use the simulated data based on $n=100, \alpha=0.8, \beta=1.3, \theta=0.5$, and $r=0.3$, and at accelerated condition, $n_{a}=10$. The MLEs of the model parameters and acceleration factor obtained by using the $\mathrm{R}$ software are $\widehat{\alpha}=1.5757, \widehat{\theta}=0.2216$, and $\widehat{\beta}=1.4198$. The inverse of the estimated Fisher information matrix is

$$
F=\left[\begin{array}{ccc}
138.4549 & 154.5262 & 24.7334 \\
154.5262 & 60.1488 & 5.7169 \\
24.73340 & 5.7169 & 20.6185
\end{array}\right]
$$

The $90 \%$ and $95 \%$ interval estimates for the model parameters and acceleration factor are provided in Table 4. 
TABLE 2: Average estimates of CSPLAT with $\alpha=1, \beta=1.1, \theta=1$, and $r=0.3$

\begin{tabular}{|c|c|c|c|c|c|}
\hline$\eta$ & Parameters & Estimates & MSE & Absolute bias & $95 \% \mathrm{CI}$ \\
\hline \multirow{3}{*}{50} & $\alpha$ & 2.1466 & 1.4601 & 1.12465 & $\begin{array}{c}-1.1840 \\
5.5040\end{array}$ \\
\hline & $\beta$ & 0.7112 & 0.46457 & 0.38876 & $\begin{array}{c}-0.9333 \\
2.2885\end{array}$ \\
\hline & $\theta$ & 0.5080 & 0.24811 & 0.49196 & $\begin{array}{c}-0.8467 \\
1.8718 \\
\end{array}$ \\
\hline \multirow{3}{*}{100} & $\alpha$ & 1.9824 & 1.04411 & 0.98242 & $\begin{array}{c}-0.8141 \\
4.7996\end{array}$ \\
\hline & $\beta$ & 0.7513 & 0.28392 & 0.34868 & $\begin{array}{c}-0.4538 \\
1.9765\end{array}$ \\
\hline & $\theta$ & 0.5161 & 0.23707 & 0.48393 & $\begin{array}{c}-0.8336 \\
1.8634\end{array}$ \\
\hline \multirow{3}{*}{150} & $\alpha$ & 1.9443 & 0.94151 & 0.94430 & $\begin{array}{c}-0.7090 \\
4.5968\end{array}$ \\
\hline & $\beta$ & 0.8099 & 0.23370 & 0.29013 & $\begin{array}{c}-0.2890 \\
1.9258\end{array}$ \\
\hline & $\theta$ & 0.5156 & 0.23648 & 0.48444 & $\begin{array}{c}-0.8350 \\
1.8635\end{array}$ \\
\hline \multirow{3}{*}{200} & $\alpha$ & 1.9318 & 0.90238 & 0.93182 & $\begin{array}{c}-0.6499 \\
4.4828\end{array}$ \\
\hline & $\beta$ & 0.8066 & 0.21864 & 0.29344 & $\begin{array}{c}-0.2114 \\
1.9076\end{array}$ \\
\hline & $\theta$ & 0.5160 & 0.23574 & 0.48405 & $\begin{array}{c}-0.8345 \\
1.8628 \\
\end{array}$ \\
\hline \multirow{3}{*}{300} & $\alpha$ & 1.9043 & 0.84035 & 0.90430 & $\begin{array}{c}-0.6121 \\
4.4129\end{array}$ \\
\hline & $\beta$ & 0.8315 & 0.18293 & 0.26852 & $\begin{array}{c}-0.1070 \\
1.8217\end{array}$ \\
\hline & $\theta$ & 0.5166 & 0.23464 & 0.48344 & $\begin{array}{c}-0.8333, \\
1.8619 \\
\end{array}$ \\
\hline \multirow{3}{*}{500} & $\alpha$ & 1.8991 & 0.82207 & 0.89914 & $\begin{array}{c}-0.5948 \\
4.3840\end{array}$ \\
\hline & $\beta$ & 0.8488 & 0.16980 & 0.25116 & $\begin{array}{c}-0.0358 \\
1.7549\end{array}$ \\
\hline & $\theta$ & 0.5145 & 0.23628 & 0.48553 & $\begin{array}{c}-0.8374, \\
1.8635\end{array}$ \\
\hline
\end{tabular}
are

The asymptotic variance-covariance matrix of $\alpha, \theta$, and $\beta$

$$
V=\left[\begin{array}{lll}
0.0072 & 0.0065 & 0.0404 \\
0.0065 & 0.0166 & 0.1749 \\
0.0404 & 0.1749 & 0.0485
\end{array}\right]
$$

The $95 \%$ asymptotic confidence interval for $\alpha, \theta$, and $\beta$ are presented, respectively:

$$
\begin{gathered}
1.4093 \leq \alpha \leq 1.7420, \\
-0.0309 \leq \theta \leq 0.4741, \\
0.9881 \leq \beta \leq 1.8514 .
\end{gathered}
$$

From the results, we observe that the point estimates are stable. We also examine that the results support theoretical

\begin{tabular}{|c|c|c|c|c|c|}
\hline$\eta$ & Parameters & Estimates & MSE & $\begin{array}{l}\text { Absolute } \\
\text { bias }\end{array}$ & $95 \%$ CI \\
\hline \multirow{3}{*}{50} & $\alpha$ & 1.7092 & 0.9542 & 0.9092 & \multirow{3}{*}{$\begin{array}{c}-0.9064 \\
4.3248 \\
0.3143,2.0921 \\
-0.9225 \\
1.6123\end{array}$} \\
\hline & $\beta$ & 1.2032 & 0.2057 & 0.0032 & \\
\hline & $\theta$ & 0.3449 & 0.2111 & 0.4551 & \\
\hline \multirow{3}{*}{100} & $\alpha$ & 1.5806 & 0.6579 & 0.7806 & \multirow{3}{*}{$\begin{array}{c}-0.6258 \\
3.7871 \\
0.5454,1.9059 \\
-0.8932 \\
1.5971\end{array}$} \\
\hline & $\beta$ & 1.2256 & 0.1198 & 0.0257 & \\
\hline & $\theta$ & 0.3519 & 0.2028 & 0.4480 & \\
\hline \multirow{3}{*}{150} & $\alpha$ & 1.5592 & 0.6060 & 0.7592 & \multirow{3}{*}{$\begin{array}{c}-0.5721 \\
3.6905 \\
0.6059,1.9067 \\
-0.9124 \\
1.6055 \\
\end{array}$} \\
\hline & $\beta$ & 1.2563 & 0.1070 & 0.0563 & \\
\hline & $\theta$ & 0.3465 & 0.2070 & 0.4535 & \\
\hline \multirow{3}{*}{200} & $\alpha$ & 1.5407 & 0.5723 & 0.7407 & \multirow{3}{*}{$\begin{array}{c}-0.5344 \\
3.6158 \\
0.6928,1.8614 \\
-0.8998 \\
1.5994\end{array}$} \\
\hline & $\beta$ & 1.2771 & 0.0829 & 0.0772 & \\
\hline & $\theta$ & 0.3498 & 0.2058 & 0.4502 & \\
\hline \multirow{3}{*}{300} & $\alpha$ & 1.5204 & 0.5324 & 0.7203 & \multirow{3}{*}{$\begin{array}{c}-0.4893 \\
3.5301 \\
0.7487,1.8324 \\
-0.9058 \\
1.6019\end{array}$} \\
\hline & $\beta$ & 1.2906 & 0.0682 & 0.0905 & \\
\hline & $\theta$ & 0.3481 & 0.2049 & 0.4519 & \\
\hline \multirow{3}{*}{500} & $\alpha$ & 1.5081 & 0.5092 & 0.7081 & \multirow{3}{*}{$\begin{array}{c}-0.4623 \\
3.4784 \\
0.8045,1.7806 \\
-0.9103 \\
1.6038\end{array}$} \\
\hline & $\beta$ & 1.2925 & 0.0534 & 0.0926 & \\
\hline & $\theta$ & 0.3467 & 0.2040 & 0.4502 & \\
\hline
\end{tabular}
findings of CSPALT for Fréchet distribution.
TABLE 3: Average estimates of CSPLAT with $\alpha=0.8, \beta=1.2, \theta=0.8$, and $r=0.3$

TABLE 4: Interval estimates of the parameters.

\begin{tabular}{lcc}
\hline Parameters & $90 \%$ CI & $95 \%$ CI \\
\hline$\alpha$ & $-0.6142,3.7657$ & $-0.2623,3.4137$ \\
$\beta$ & $0.26319,2.5764$ & $0.4491,2.3905$ \\
$\theta$ & $-0.5521,0.9953$ & $-0.4277,0.8710$ \\
\hline
\end{tabular}

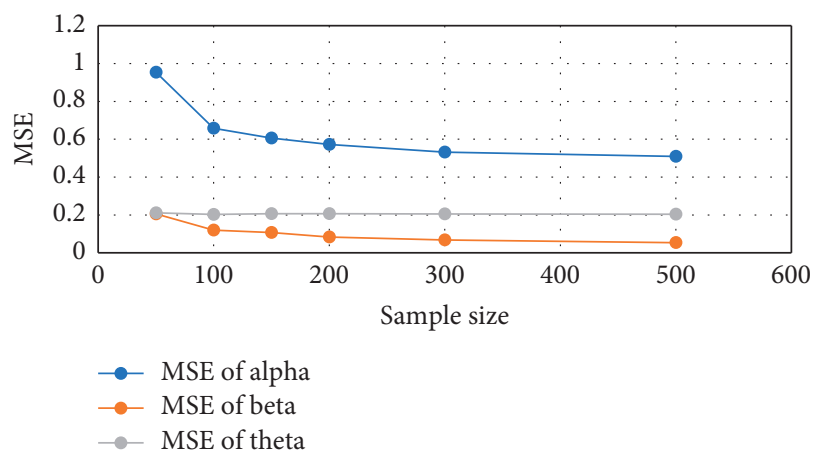

FIgURE 1: Graph of MSE versus sample size when $\alpha=0.75, \beta=1.2$, and $\theta=1$. 


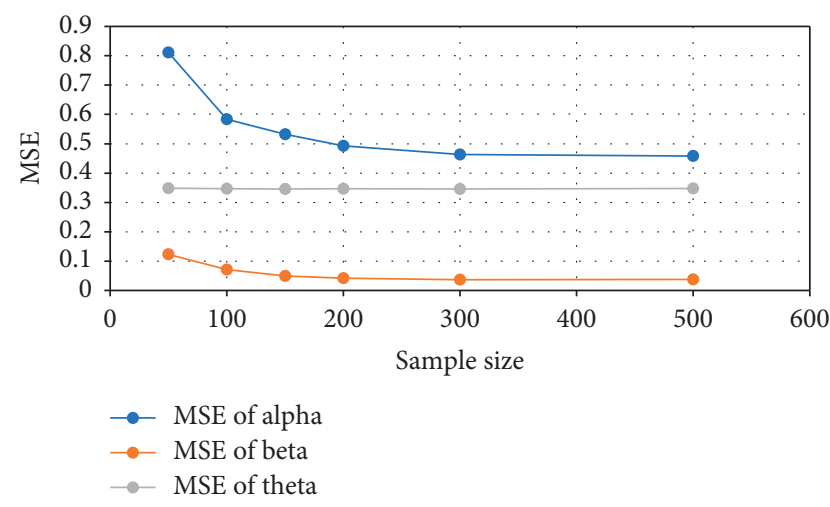

FIGURE 2: Graph of MSE versus sample size when $\alpha=1, \beta=1.1$, and $\theta=1$.

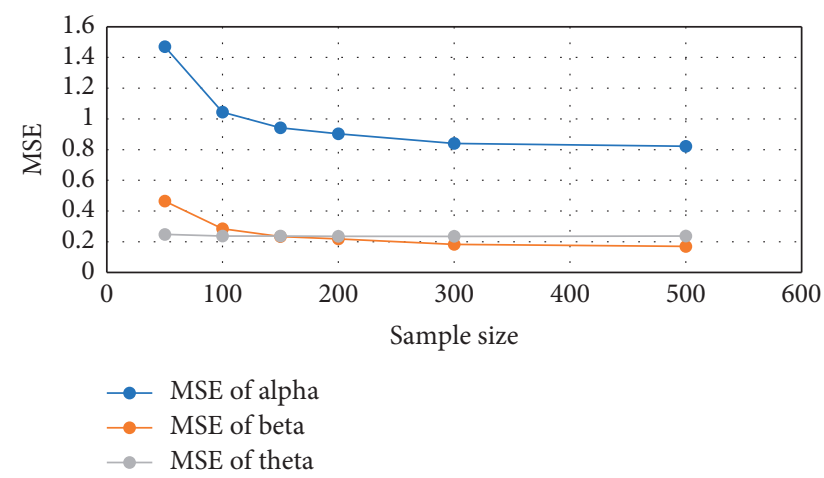

FIGURE 3: Graph of MSE versus sample size when $\alpha=0.8, \beta=1.2$, and $\theta=0.8$.

\section{Conclusion}

From Tables 1-3, it is observed that the MLEs converge to the true values of the parameters with an increase in sample size " $n$." Also, we find that the MLEs have good statistical properties for a fixed value of parameters, as we increase sample size, the MSE and bias of the estimators are decreased. Moreover, from Tables $1-3$, the MSEs of $\alpha$ decrease as we increase the sample size. It can be seen from results in Tables 1-3 that when there is an increment in sample size, the interval of the estimates decreases. The outcomes of simulation suggest that PALT is an appropriate model using Fréchet distribution which enables us to save cost and time without using a higher level of stress to all test items. From simulation results, it is concluded that the MLEs have good statistical properties.

\section{Recommendation}

Some pertinent suggestions are also given for future research which is tantamount to provide a pathway for future researchers in the field of PALT. This work can be extended for type-II censoring of CSPALT using Fréchet distribution.

\section{Data Availability}

The data used in this article are freely available upon request from the authors and citing this paper in your manuscripts.

\section{Conflicts of Interest}

The authors declare that they have no conflicts of interest to report regarding the present study.

\section{References}

[1] C. A. Lloyd, T. Roulstone, and R. E. Lyons, "Transport, constructability, and economic advantages of SMR modularization," Progress in Nuclear Energy, vol. 134, p. 103672, 2021.

[2] X. Shi, P. Lu, and Y. Shi, "Reliability estimation for hybrid system under constant-stress partially accelerated life test with progressively hybrid censoring," Recent Patents on Engineering, vol. 14, no. 1, pp. 82-94, 2020.

[3] V. S. Yakovyna, M. M. Seniv, I. I. Symets, and N. B. Sambir, "Algorithms and software suite for reliability assessment of complex technical systems," Radio Electronics, Computer Science, Control, vol. 4, pp. 163-177, 2020.

[4] A. Abd El-Raheem, "Optimal plans and estimation of constant-stress accelerated life tests for the extension of the exponential distribution under type-I censoring," Journal of Testing and Evaluation, vol. 47, no. 5, pp. 3781-3821, 2018.

[5] H. M. Aljohani and N. M. Alfar, "Estimations with step-stress partially accelerated life tests for competing risks Burr XII lifetime model under type-II censored data," Alexandria Engineering Journal, vol. 59, no. 3, pp. 1171-1180, 2020.

[6] S. Dey and M. Nassar, "Classical methods of estimation on constant stress accelerated life tests under exponentiated Lindley distribution," Journal of Applied Statistics, vol. 47, no. 6, pp. 975-996, 2020.

[7] M. M. M. El-Din, S. E. Abu-Youssef, N. S. A. Ali, and A. M. A. El-Raheem, "Estimation in constant-stress accelerated life tests for extension of the exponential distribution under progressive censoring," Metron, vol. 74, no. 2, pp. 253-273, 2016.

[8] D. S. Bai and S. W. Chung, "Optimal design of partially accelerated life tests for the exponential distribution under type-I censoring," IEEE Transactions on Reliability, vol. 41, no. 3, pp. 400-406, 1992.

[9] D. S. Bai, S. W. Chung, and Y. R. Chun, "Optimal design of partially accelerated life tests for the lognormal distribution under type I censoring," Reliability Engineering \& System Safety, vol. 40, no. 1, pp. 85-92, 1993.

[10] R. Zaman, P. Nasiri, and A. Shadrokh, "Statistical inference for the lomax distribution under partially accelerated life tests with progressively type-ii censoring with binomial removal," Jordan Journal of Mathematics and Statistics (JJMS), vol. 13, no. 3, pp. 439-458, 2020.

[11] A. A. Ismail and A. Al Tamimi, "Optimum constant-stress partially accelerated life test plans using type-I censored data from the inverse Weibull distribution," Strength of Materials, vol. 49, no. 6, pp. 847-855, 2017.

[12] A. S. Hassan, S. M. Assar, and A. N. Zaky, "Constant-stress partially accelerated life tests for inverted Weibull distribution with multiple censored data," International Journal of Advanced Statistics and Probability, vol. 3, no. 1, pp. 72-82, 2015.

[13] M. Kamal, S. Zarrin, and A. Islam, "Constant stress partially accelerated life test design for inverted Weibull distribution with type-I censoring," Algorithms Research, vol. 2, no. 2, pp. 43-49, 2013.

[14] A. M. Abd-Elfattah, A. S. Hassan, and S. G. Nassr, "Estimation in step-stress partially accelerated life tests for the Burr type XII distribution using type I censoring," Statistical Methodology, vol. 5, no. 6, pp. 502-514, 2008. 
[15] I. Alam and A. Ahmed, "Parametric and interval estimation under step-stress partially accelerated life tests using adaptive type-II progressive hybrid censoring," Annals of Data Science, pp. 1-13, 2020.

[16] Ç. Çetinkaya, "Estimation in step-stress partially accelerated life tests for the power lindley distribution under progressive censoring," Gazi University Journal of Science, 2021.

[17] T. A. Abushal and A. A. Soliman, "Estimating the Pareto parameters under progressive censoring data for constantpartially accelerated life tests," Journal of Statistical Computation and Simulation, vol. 85, no. 5, pp. 917-934, 2015.

[18] D. Han and T. Bai, "On the maximum likelihood estimation for progressively censored lifetimes from constant-stress and step-stress accelerated tests," Electronic Journal of Applied Statistical Analysis, vol. 12, no. 2, pp. 392-404, 2019.

[19] G. Prakash, "Bayes estimation in step-stress PALT on type-I progressive hybrid Rayleigh data," Afrika Statistika, vol. 14, no. 4, pp. 2165-2178, 2019.

[20] A. Ismail, The Test Design and Parameter Estimation of Pareto Lifetime Distribution under Partially Accelerated Life Tests, Cairo University, Giza, Egypt, 2004.

[21] A. A. Ismail, A. A. Abdel-Ghaly, and E. H. El-Khodary, "Optimum constant-stress life test plans for Pareto distribution under type-I censoring," Journal of Statistical Computation and Simulation, vol. 81, no. 12, pp. 1835-1845, 2011.

[22] Y.-F. Cheng and F.-K. Wang, "Estimating the Burr XII parameters in constant-stress partially accelerated life tests under multiple censored data," Communications in Statistics-Simulation and Computation, vol. 41, no. 9, pp. 17111727, 2012.

[23] A. A. Ismail, "Estimating the parameters of Weibull distribution and the acceleration factor from hybrid partially accelerated life test," Applied Mathematical Modelling, vol. 36, no. 7, pp. 2920-2925, 2012.

[24] L. Wang, "Estimation of constant-stress accelerated life test for Weibull distribution with nonconstant shape parameter," Journal of Computational and Applied Mathematics, vol. 343, pp. 539-555, 2018.

[25] L. Wang and Y. Shi, "Estimation for constant-stress accelerated life test from generalized half-normal distribution," Journal of Systems Engineering and Electronics, vol. 28, no. 4, pp. 810-816, 2017.

[26] M. Kumar, A. Pathak, and S. Soni, "Bayesian inference for Rayleigh distribution under step-stress partially accelerated test with progressive type-II censoring with binomial removal," Annals of Data Science, vol. 6, no. 1, pp. 117-152, 2019.

[27] G. Prakash, "Bayes estimation under different censoring patterns on constant-stress PALT," Austrian Journal of Statistics, vol. 47, no. 4, pp. 60-74, 2018.

[28] S. Roy, "Bayesian accelerated life test plans for series systems with Weibull component lifetimes," Applied Mathematical Modelling, vol. 62, pp. 383-403, 2018.

[29] Y. Wu, S. Younas, K. Abbas, A. Ali, and S. A. Khan, "Monitoring reliability for three-parameter Frechet distribution using control charts," IEEE Access, vol. 8, pp. 7124571253, 2020.

[30] M. Bladt, L. J. R. Esparza, and B. F. Nielsen, "Fisher information and statistical inference for phase-type distributions," Journal of Applied Probability, vol. 48, pp. 277-293, 2011. 